\title{
Diagnostic Accuracy of Unenhanced Abbreviated Diffusion-Weighted Magnetic Resonance Imaging Versus Postcontrast Abbreviated Breast Magnetic Resonance Imaging for Breast Cancer
}

\author{
YJ Kim \\ Department of Radiology, Konyang University Hospital, Republic of Korea
}

\begin{abstract}
Objectives: The purpose of this study was to compare the detectability of breast cancer by unenhanced abbreviated magnetic resonance imaging (MRI) based on diffusion-weighted imaging (DWI) with the detectability of breast cancer by postcontrast abbreviated MRI.

Methods: Between January 2014 and December 2015, a total of 89 patients were enrolled. Bilateral breast cancers were found in three patients, for a total of 92 breasts with breast cancer and 86 negative breasts. All breast MRIs were performed using a 3T MRI scanner with a 7-channel radiofrequency coil. Postcontrast abbreviated MRI consisted of T2-weighted images (T2WI), TI-weighted images (TIWI), and a postcontrast TIWI sequence. Unenhanced abbreviated MRI included T2WI, TIWI, and DWI. Qualitative and quantitative analyses of the apparent diffusion coefficient map were performed independently. The sensitivity and specificity were calculated. Receiver operating characteristic analysis was performed and the areas under the curves (AUCs) were compared between these protocols. We evaluated factors associated with false negativity.

Results: The sensitivity/specificity of postcontrast abbreviated MRI, qualitative and quantitative analyses of abbreviated DWI MRI were $94.6 \% / 94.2 \%, 84.8 \% / 97.7 \%$, and $87.0 \% / 98.8 \%$, respectively. The AUCs for the postcontrast abbreviated MRI, and qualitative and quantitative analyses of DWI MRI were 0.944, 0.912, and 0.929, respectively $(p>0.05)$. The false-negative rate of unenhanced abbreviated MRI was higher than that of postcontrast abbreviated MRI without significant $(p>0.05)$. Smaller cancers $(\leq 10 \mathrm{~mm})$ was associated false negativity.

Conclusion: The diagnostic performance of abbreviated DWI breast MRI was comparable to that of postcontrast abbreviated MRI.
\end{abstract}

Key Words: Breast; Diagnosis; Magnetic resonance imaging; Neoplasms Correspondence: Dr YJ Kim, Department of Radiology, Konyang University Hospital, Republic of Korea
Email: jjoong0318@gmail.com

Submitted: 12 Mar 2020; Accepted: 18 May 2020

Contributors: The author designed the study, acquired the data, analysed the data, drafted the manuscript, and critically revised the manuscript for important intellectual content. The author had full access to the data, contributed to the study, approved the final version for publication, and takes responsibility for its accuracy and integrity.

Conflicts of Interest: The author declared that no conflicts of interest.

Funding/Support: This research received no specific grant from any funding agency in the public, commercial, or not-for-profit sectors.

Data Availability: All data generated or analysed during the present study are available from the corresponding author on reasonable request.

Ethics Approval: This study was approved by the institutional review board of Konyang University Hospital (Ref: 2019-12-024). 


\title{
中文摘要
}

\section{彌散加權無顯影劑加強快速磁共振成像與顯影後快速磁共振成像對 乳腺癌的診斷準確性比較}

\author{
YJ Kim \\ 目的 : 本研究旨在比較彌散加權 (DWI) 無顯影劑加強快速磁共振成像 (MRI) 與顯影後快速MRI \\ 對乳腺癌的檢測性能。 \\ 方法：2014年1月至2015年12月期間共納入89例患者, 當中92個乳腺有乳腺癌（雙側乳腺癌3例）, \\ 86個乳腺為陰性。所有乳腺MRI均使用7通道射頻線圈的3T MRI掃描儀。顯影後快速MRI包括T2加 \\ 權圖像（T2WI）、T1加權圖像（T1WI）及顯影後T1WI序列。無顯影劑加強快速MRI包括 T2WI、 \\ T1WI和DWI 獨立進行表觀擴散系數圖的定性和定量分析並計算敏感性和特異性。進行接受者操作 \\ 特徵分析並比較這些方案之間的曲線下面積 (AUC)。評估與假陰性相關的因素。 \\ 結果：顯影後快速MRI、DWI MRI的定性及定量分析的敏感性與特異性分別為 $94.6 \% / 94.2 \%$ 、 \\ $84.8 \% / 97.7 \%$ 和 $87.0 \% / 98.8 \%$ 。顯影後快速MRI、DWI MRI的定性及定量分析的AUC分別為 0.944 、 \\ 0.912 和 $0.929(\mathrm{p}>0.05)$ 。無顯影劑加強快速MRI的假陰性率高於顯影後快速MRI但統計不顯著 \\ （ $\mathrm{p}>0.05 ）$ 。腫瘤較細小（ $\leq 10$ 毫米）與假陰性相關。 \\ 結論 : 乳腺DWI快速MRI與顯影後快速MRI的診斷表現相似。
}

\section{INTRODUCTION}

Breast magnetic resonance imaging (MRI) has a higher sensitivity than digital mammography in women with a familial or genetic predisposition. ${ }^{1-3}$ Screening by breast MRI is recommended for women with a personal history or a high-risk lesion. ${ }^{4}$ However, a full diagnostic protocol (FDP) of breast MRI has drawbacks, including high cost, lengthy acquisition time (mean time, 24 min; range, 17-40 $\mathrm{min}$ ), and use of intravenous contrast. ${ }^{5}$

Abbreviated postcontrast breast MRI examinations have been proposed as an alternative method; they typically include only a precontrast sequence and an early-phase postcontrast sequence with/without T2-weighted imaging (T2WI). ${ }^{5-10}$ The mean acquisition time of these shortened examinations is $9 \mathrm{~min}$ (range, $3-15 \mathrm{~min}$ ). ${ }^{11}$ Abbreviated postcontrast MRI scans have been reported to provide cancer detection rates and a diagnostic accuracy that are equivalent to those provided by an FDP. ${ }^{5-10}$ These results are promising for the development of screening MRI protocols that are more efficient for women at high risk of breast cancer.

Current abbreviated breast MRI protocols still involve administration of intravenous gadolinium contrast material. Intravenous contrast may increase the cost, the examination time, or incidence of adverse effects. In addition, gadolinium contrast is contraindicated in patients with kidney disease or contrast allergy. These are considerations for an asymptomatic young population with indications for breast cancer screening. ${ }^{11,12}$

Diffusion-weighted imaging (DWI), which measures endogenous water movement within tissues, is a fast, widely available MRI technique that requires no gadolinium contrast material. The resulting apparent diffusion coefficient (ADC) values are quantified by mean diffusivity measurements in three orthogonal directions. ADC values are influenced by tissue cellularity, fluid viscosity, membrane permeability and blood flow, and are known to be useful for discriminating between benign and malignant lesions. ${ }^{13}$ Several studies have reported high yields from the combined use of DWI MRI added to FDP MRI. ${ }^{14-16}$ Recent studies distinguishing between benign and malignant lesions have reported that DWI MRI alone showed a diagnostic performance comparable to that provided by FDP MRI ${ }^{14-16}$ Kang et al ${ }^{17}$ compared the performance of DWI MRI to that of abbreviated postcontrast MRI. They reported that the performance of DWI MRI as a screening examination in patients with 
a personal history of breast cancer was comparable to that of abbreviated postcontrast MRI, with reduced times for image acquisition and interpretation. ${ }^{17}$ Few studies have investigated the feasibility of unenhanced MRI comprising T1-weighted images (T1WI), T2WI, and DWI as a screening method in populations at risk.

The purpose of this study was to compare the detectability of breast cancer by MRI based on DWI with that by postcontrast abbreviated MRI. We also compared the diagnostic performance of DWI MRI using a quantitative analysis that required ADC values and a qualitative analysis that was performed by visual assessment without ADC mapping.

\section{METHODS}

\section{Participants and Selection Process}

This retrospective study was approved by our institutional review board, and the requirement for informed consent was waived. The initial study population consisted of consecutive patients who underwent breast MRI with FDP between January 2014 and December 2015 for screening, difficult cases, evaluating the extension of breast cancer, detection of additional lesions, or response to neoadjuvant chemotherapy. Patients were excluded if they had prior surgery, had been treated with neoadjuvant chemotherapy, had MR findings that were difficult to correlate with biopsy results, had implant or silicone injection, or had previous mammotomy or stereotactic biopsy.

\section{Magnetic Resonance Imaging Techniques}

All breast MRIs were performed in a 3T MRI scanner (Achieva; Philips Healthcare, Best, the Netherlands) equipped with a 7-channel breast radiofrequency coil, with the patient in the prone position. DWI was examined by a spin-echo-type single-shot echo planar imaging technique in the axial plane. Diffusionsensitising gradients were applied in three orthogonal directions (lateral, sagittal, and craniocaudal) and images were obtained at $b$ values of 0 and $1,000 \mathrm{~s} / \mathrm{mm}^{2} .^{18,19}$ The scanning parameters are listed in Table 1 . The contrast agent (gadobutrol, $0.1 \mathrm{mmol} / \mathrm{kg}$ ) was injected into an antecubital vein using an automated injector at a rate of $1.2 \mathrm{~mL} / \mathrm{s}$, followed by a $20-\mathrm{mL}$ saline flush. We acquired subtraction images as follows: the baseline data, acquired before infusion of contrast agent, were subtracted (slice by slice, and for each slice, pixel by pixel).

\section{Image Interpretation}

All FDP MRIs were interpreted. The sequences making up the postcontrast abbreviated MRI consisted of turbospin-echo (TSE) T2WI with fat suppression, TSE-T1WI, a single intermediate (3 min after contrast injection) postcontrast $\mathrm{T} 1$ sequence, and a subtraction image. The sequences comprising unenhanced abbreviated MRI, which was based on DWI, included TSE-T2WI with fat suppression, TSE-T1WI, and DWI. All lesions were evaluated according to a checklist, which included the following items: location of the suspicious lesion, size, shape, margin, enhancement pattern, T2WI signal intensity (high, intermediate, or low), DWI detectability, ADC value, ADC signal intensity, and Breast Imaging Reporting and Data System (BI-RADS) assessment.

For postcontrast abbreviated MRI, the readers identified any suspicious findings on the postcontrast sequence, and then analysed the corresponding lesions on fatsuppressed T2WI and T1WI. For abbreviated DWI MRI, the readers detected breast lesions showing high signal intensity on DWI with the high $b$ value $\left(b=1,000 \mathrm{~s} / \mathrm{mm}^{2}\right)$, and then reviewed the fat-suppressed T2WI and T1WI. Qualitative evaluation was performed by a visual assessment of the signal intensity on DWI acquired at $\mathrm{b}=1000 \mathrm{~s} / \mathrm{mm}^{2}$ and their corresponding ADC maps. For visual evaluation, DWI detectability was classified as detectable, equivocal, or undetectable. ADC signal intensity was classified as high, low, or undetectable. A solid tumour showing high signal intensity on the high-bvalue DWI and low signal intensity on the corresponding

Table 1. Scanning parameters of full diagnostic protocols.

\begin{tabular}{|c|c|c|c|c|c|c|c|c|c|}
\hline Sequence & Orientation & $\begin{array}{l}\mathrm{TR} / \mathrm{TE} \\
(\mathrm{ms})\end{array}$ & FA & $\begin{array}{c}\text { Fat } \\
\text { suppression }\end{array}$ & $\mathrm{FOV}(\mathrm{mm})$ & $\begin{array}{l}\text { Resolution } \\
\quad(\mathrm{mm})\end{array}$ & $\begin{array}{l}\text { Thickness } \\
\text { (mm) }\end{array}$ & $\begin{array}{l}\text { No. of signals } \\
\text { acquired }\end{array}$ & $\begin{array}{l}\text { Scan } \\
\text { time }(s)\end{array}$ \\
\hline T1-weighted TSE & Transverse & $4.1 / 2.1$ & 12 & SPAIR & $340 \times 340$ & $484 \times 482$ & 3 & 1 & 162 \\
\hline T2-weighted TSE & Transverse & $2660 / 70$ & 90 & SPAIR & $339 \times 339$ & $452 \times 442$ & 4 & 1 & 245 \\
\hline DWI echo planar imaging & Transverse & $6907 / 66$ & 90 & SPAIR & $360 \times 360$ & $132 \times 128$ & 4 & 4 & 400 \\
\hline $\begin{array}{l}\text { Three-dimensional dynamic } \\
\text { contrast enhancement TSE }\end{array}$ & Transverse & 4.1/2.1 & 12 & SPAIR & $340 \times 340$ & $484 \times 482$ & 3 & 1 & 60 \\
\hline
\end{tabular}

Abbreviations: FA = flip angle; FOV = field of view; SPAIR = spectral adiabatic inversion recovery; TR/TE = repetition time/echo time; TSE = turbo-spin-echo. 
ADC map was considered to require histopathological confirmation. We performed quantitative evaluations, using the CAD system (CADstream version 6.0; Confirma, Kirkland [WA], United States) to identify ADC values corresponding to the lesions detected on visual assessment. The ADC map of the largest diameter of each tumour was selected, and a region of interest was manually drawn to encompass the entire cross-section of the lesion avoiding adjacent normal breast tissue or fat. Cystic, necrotic, or haemorrhagic components of the lesions that might affect the ADC values were also avoided. The ADC cut-off was set as $1.25 \times 10^{-3} \mathrm{~mm}^{2} / \mathrm{s}^{20,21}$ In this study, any lesion with an ADC value lower than the cut-off value was considered to require histopathological confirmation. Visual assessments and quantitative analyses for ADC map were performed independently. We assessed background intensity for DWI. There is no definition of background intensity for DWI MRI in BIRADS; however, we applied a definition similar to that of BI-RADS. The degree of background diffusion signals on DWI was visually assessed and graded as minimal, mild, moderate and severe. BI-RADS final assessment categories 1, 2, and 3 were considered MRI-negative, and categories 4 and 5 were considered MRI-positive. Finally, the readers were instructed to record the length of time needed to interpret each case. These image evaluation sessions were assessed at 2 -week intervals.

\section{Histopathological Evaluation}

The histopathological diagnoses were retrieved from the electronic records of our institution. The final histopathological diagnoses were made based on evaluations of the surgical specimens of patients who underwent surgery after the imaging studies. Evaluation of core needle biopsy specimens was considered representative for patients who refused surgery or who were transferred to other hospitals.

The intrinsic lesion subtype was determined based on the following markers: expression of oestrogen receptor, progesterone receptor, and human epidermal growth factor receptor 2 (HER2). Tumours with oestrogen receptor and/or progesterone receptor positivity were defined as having $\geq 10 \%$ of tumour cells with positive nuclei. HER2 expression was evaluated by the HercepTest (Dako, Glostrup, Denmark) and scored on a scale from 0 to $3+$. Tumours with scores $\geq 3$ or with a $\geq 2$.2-fold increase in HER2 gene amplification, as determined by fluorescence in situ hybridisation, were considered positive for HER2 overexpression.

\section{Statistical Analysis}

Sensitivity and specificity were calculated. Receiver operating characteristic curve analysis was performed, and the areas under the curves were compared between protocols. The Chi-square or Fisher's exact test was used to evaluate false negativity according to tumour size, histopathological results, and background intensity on DWI. The reading times for both protocols were assessed by paired $t$ tests. Statistical analysis was performed by SPSS (Windows version 18.0; SPSS Inc., Chicago [IL], United States). A p value of $<0.05$ was considered significant.

\section{RESULTS Patients}

In total, 175 consecutive patients underwent breast MRI with FDP between January 2014 and December 2015 for screening, difficult cases, evaluating the extension of breast cancer, detection of additional lesions, or response to neoadjuvant chemotherapy (Figure 1). A total of 86 patients were excluded. For patients with multicentric cancers, the largest tumour was considered as the representative. Bilateral breast cancers were found in three patients. Each lesion was counted as one lesion. A total of 89 patients were enrolled, with 92 breasts with breast cancer and 86 negative breasts.

\section{Histopathological Evaluation}

The histopathological diagnosis was obtained on a surgical specimen in 65 patients and a core needle biopsy

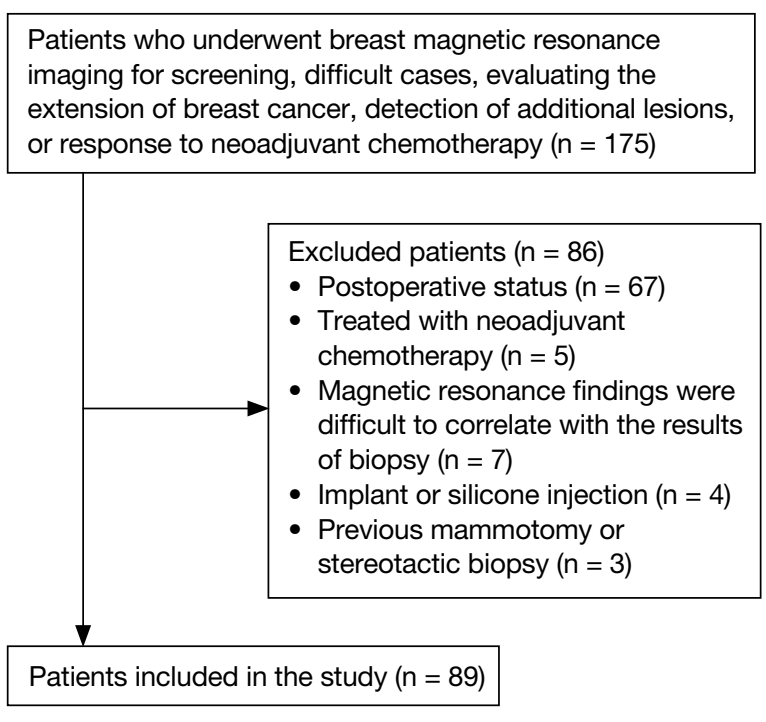

Figure 1. Patient enrolment. 
specimen in 24 patients. A total of 92 breast cancer lesions were detected. The mean tumour size was $2.55 \pm 1.59 \mathrm{~cm}$ (range, 0.3-9.0). The histological types of the breast cancers are shown in Table 2. Invasive ductal carcinoma (IDC) and ductal carcinoma in situ (DCIS) accounted for $92.4 \%$ of the cancer lesions. Most of the lesions were of the luminal B histological subtype, and $21.7 \%$ were either HER2-overexpressing or triple-negative subtypes (Table 2).

\section{Performance of Abbreviated Protocols}

The sensitivity and specificity of postcontrast abbreviated MRI were $94.6 \%$ (87/92) and $94.2 \%$ (81/86), respectively. For DWI MRI, the sensitivity and specificity of qualitative and quantitative analysis were as follows: qualitative, $84.8 \%$ (78/92), $97.7 \%$ (84/86), respectively; and quantitative, $87.0 \%$ (80/92), $98.8 \%$ (85/86), respectively. The differences between the diagnostic accuracy of these protocols were not significant (Table 3).

Table 2. Breast cancer histological types and subtypes $(n=92)$.

\begin{tabular}{lc}
\hline & No. (\%) \\
\hline Histological types & \\
Invasive ductal carcinoma & $36(39.1 \%)$ \\
Invasive ductal carcinoma and DCIS & $31(33.7 \%)$ \\
DCIS & $18(19.6 \%)$ \\
Invasive lobular carcinoma & $2(2.2 \%)$ \\
Mucinous carcinoma & $2(2.2 \%)$ \\
Invasive papillary carcinoma & $2(2.2 \%)$ \\
Adenoid cystic carcinoma & $1(1.1 \%)$ \\
Subtypes & \\
Luminal A & $22(23.9 \%)$ \\
Luminal B & $50(54.3 \%)$ \\
HER 2 & $8(8.7 \%)$ \\
Triple-negative & $12(13.0 \%)$ \\
\hline Abbreviations: DCIS = ductal carcinoma in situ; HER2 $=$ human \\
epidermal growth factor receptor 2.
\end{tabular}

\section{Missed Tumours}

Most of the lesions were detected with these protocols. The tumour diameters ranged from 0.6 to $7.0 \mathrm{~cm}$ (mean $=2.25 \pm 1.3 \mathrm{~cm}$ ) on postcontrast abbreviated MRI and 0.6 to $7.0 \mathrm{~cm}$ (mean $=1.98 \pm 1.1 \mathrm{~cm})$ on unenhanced abbreviated MRI. In addition, 10 lesions with diameters $\leq 1.0 \mathrm{~cm}$ were detected on unenhanced abbreviated MRI.

A total of 14 tumours were not detected by abbreviated MRI. None of the tumours was missed by postcontrast abbreviated MRI only. Of these tumours, five were missed by both postcontrast and abbreviated DWI MRI (mean diameter $1.0 \pm 0.5 \mathrm{~cm}, 0.3-1.5 \mathrm{~cm}$ ) [Figure 2]. Of the five undetected tumours, three had diameters $\leq 1 \mathrm{~cm}$. The histopathological findings of these undetected tumours were as follows: IDC ( $n=3)$, DCIS $(n=1)$, and IDC with DCIS $(n=1)$. Three tumours were luminal A and two were luminal B. Two IDC lesions were grade 1 .

The 14 tumours missed on abbreviated DWI MRI with qualitative analysis ranged from 0.3 to $5.0 \mathrm{~cm}$ (mean $1.8 \pm$ $1.3 \mathrm{~cm}$ ) in diameter. Five of these tumours had diameters $\leq 1.0 \mathrm{~cm}$. The 12 tumours missed on quantitative analysis ranged from 0.3 to $5.0 \mathrm{~cm}$ (mean $1.86 \pm 1.4 \mathrm{~cm}$ ) in diameter. Four of these tumours had diameters $\leq 1.0 \mathrm{~cm}$. A significantly higher proportion of missed breast cancers had diameters $\leq 1.0 \mathrm{~cm}(\mathrm{p}=0.006)$. The histopathological findings of these tumours undetected by abbreviated DWI MRI were as follows: mucinous carcinoma $(n=1)$, IDC $(n=4)$, DCIS $(n=5)$, and IDC with DCIS $(n=4)$ [Figure 3]. Seven tumours were luminal $A$ and seven were luminal B. In tumours missed on DWI MRI, there was no significant difference according to histopathological results and lesion subtype $(\mathrm{p}>0.05)$. The background intensities of the breasts with missed tumours on DWI MRI were as follows: minimal $(n=2)$, mild $(n=3)$, moderate $(n=4)$, and severe $(n=5)$. The differences in tumour detection according to background intensity on DWI MRI were not significant $(\mathrm{p}=0.198)$.

Table 3. Diagnostic ability and reading times for each reader according to protocol. ${ }^{\star}$

\begin{tabular}{|c|c|c|c|}
\hline & Postcontrast abbreviated MRI & $\begin{array}{l}\text { Qualitative analysis of unenhanced } \\
\text { abbreviated MRI }\end{array}$ & $\begin{array}{l}\text { Quantitative analysis of unenhanced } \\
\text { abbreviated MRI }\end{array}$ \\
\hline Sensitivity $(n=92)$ & $87(94.6 \%)$ & $78(84.8 \%)$ & $80(87.0 \%)$ \\
\hline Specificity $(n=86)$ & $81(94.2 \%)$ & $84(97.7 \%)$ & $85(98.8 \%)$ \\
\hline $\mathrm{AUC}^{+}$ & $0.944(95 \% \mathrm{Cl}=0.899-0.973)$ & $0.912(95 \% \mathrm{Cl}=0.861-0.949)$ & $0.929(95 \% \mathrm{Cl}=0.881-0.962)$ \\
\hline Reading time, $\mathrm{s}^{\ddagger}$ & $86.0 \pm 2.8$ & $32.4 \pm 1.3$ & $65.5 \pm 2.5$ \\
\hline
\end{tabular}



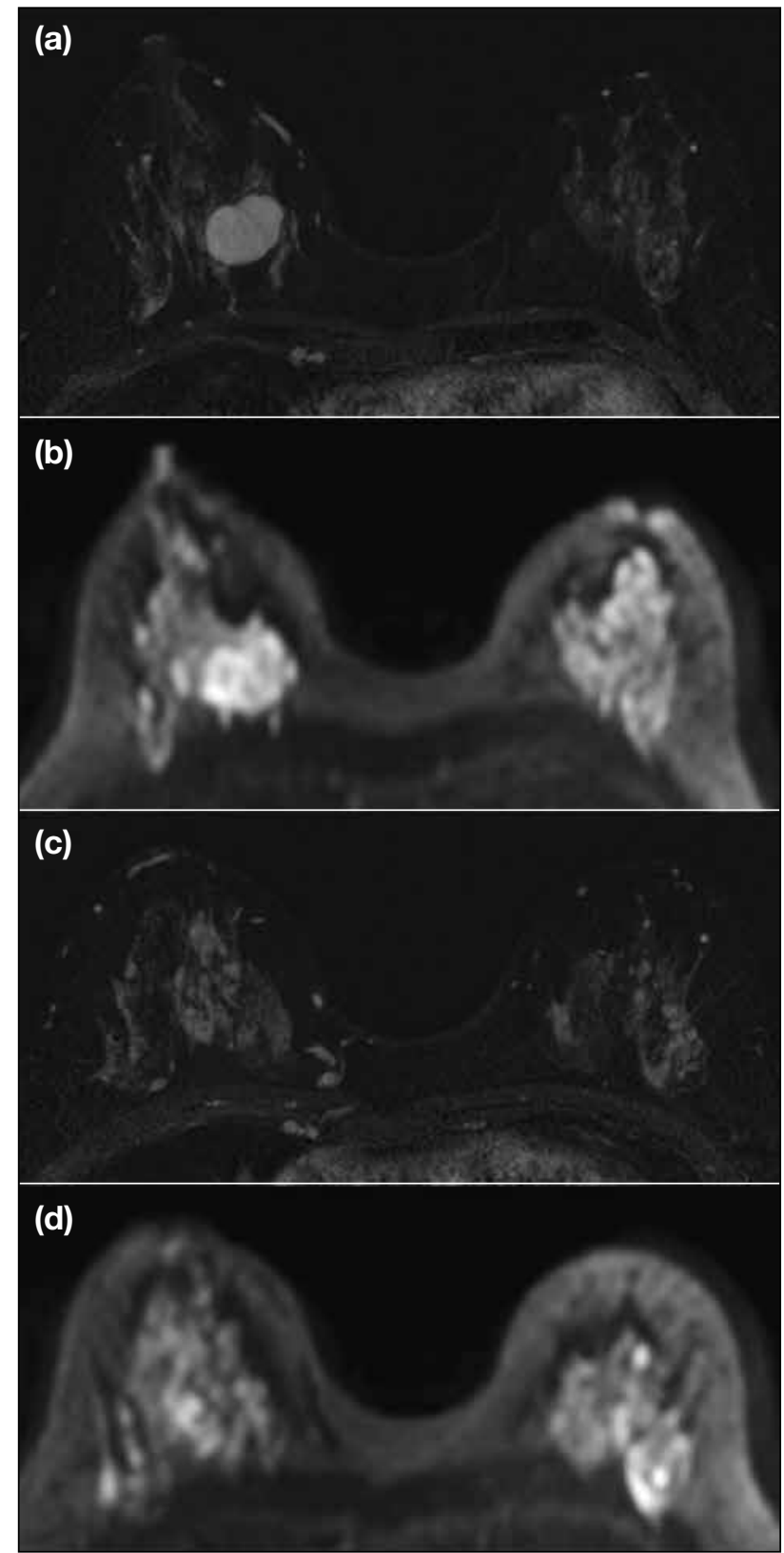

Figure 2. False-negative case. A 61-year-old woman with invasive ductal carcinoma $(0.6 \mathrm{~cm}$ in size) in the right upper inner quadrant. The tumour was missed in both postcontrast and unenhanced abbreviated magnetic resonance imaging. There was a large fibroadenoma in the right upper inner breast on (a) the subtraction image and (b) diffusion-weighted imaging (DWI). We were not able to detect any other suspicious findings in the right upper inner quadrant on (c) the subtraction image and (d) DWI.

\section{Reading Times}

The postcontrast abbreviated MRI reading time was significantly longer than that of unenhanced abbreviated MRI $(p=0.000)($ Table 3$)$.
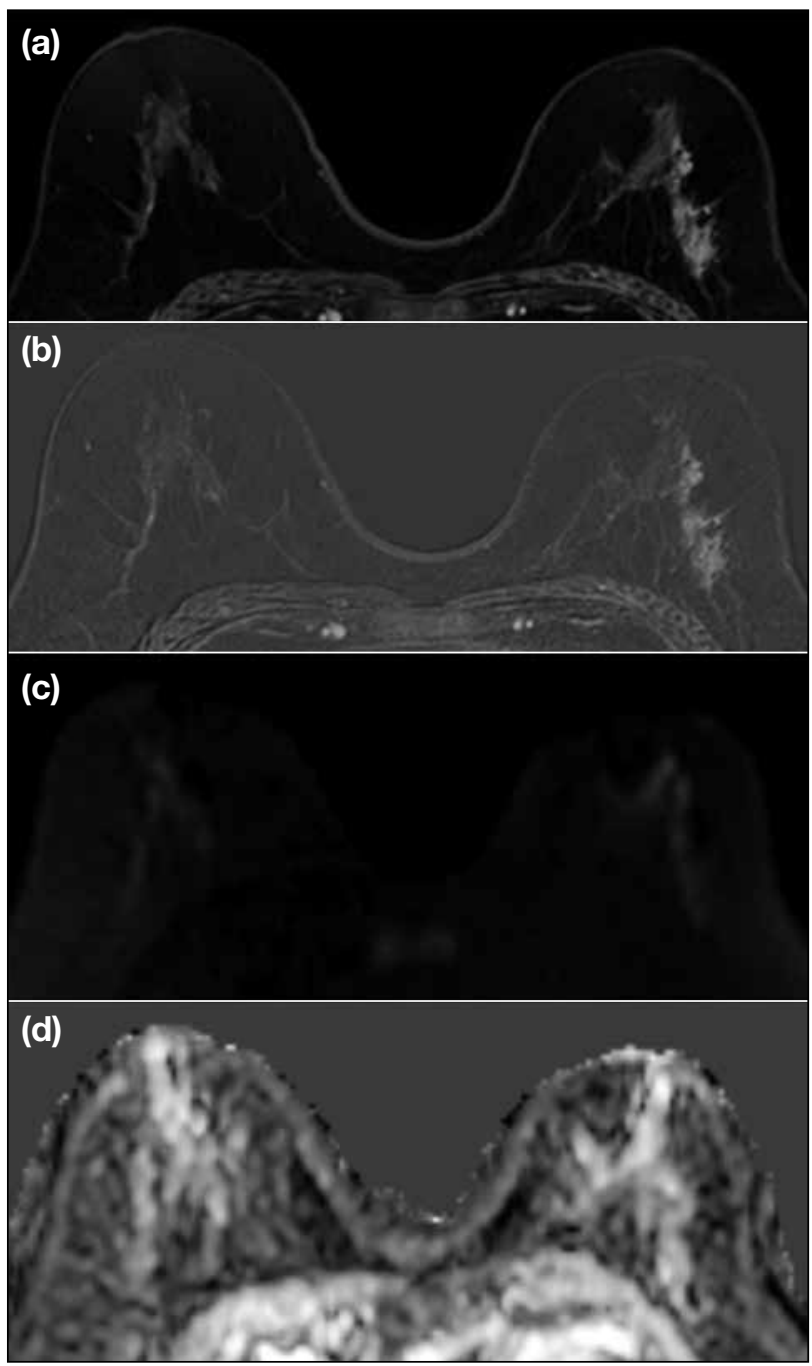

Figure 3. A 65-year-old woman with ductal carcinoma in situ in the left upper outer quadrant detected by contrast enhancement. The lesion was missed on unenhanced abbreviated magnetic resonance imaging. There was segmental non-mass enhancement in the left upper outer quadrant on (a) the postcontrast image and (b) subtraction image. We were not able to detect any suspicious findings on (c) diffusion-weighted imaging or (d) the apparent diffusion coefficient map.

\section{DISCUSSION}

We compared the diagnostic performance of unenhanced abbreviated breast MRI based on DWI with that of postcontrast abbreviated MRI for breast cancer. We found that the diagnostic performance of unenhanced abbreviated breast MRI, including qualitative and quantitative analysis, was comparable to that of postcontrast abbreviated MRI.

Postcontrast abbreviated MRI showed high sensitivity (94.6\%) in this study, which is consistent with previous studies (sensitivity ranging from $86 \%$ to $92 \%$ ). ${ }^{69,22}$ 
Those studies demonstrated that the diagnostic accuracy of postcontrast abbreviated breast MRI was similar to that of a comprehensive diagnostic MRI protocol. , $^{5,-10,22}$ Based on these results, postcontrast abbreviated breast MRI has been proposed as a promising screening tool for women at high risk of breast cancer. ${ }^{7,10,23}$

Unenhanced DWI MRI was recently introduced as an alternative methodology that avoids the risks and cost of contrast material. Several studies have compared the performance of abbreviated or standard DWI MRI to that of postcontrast abbreviated MRI. The performance of DWI MRI was reported to be equivalent to the performance of postcontrast abbreviated MRI, with reduction in image acquisition time and interpretation time for each case. ${ }^{17,24,25}$ In DWI MRI, qualitative analysis is based on visual assessment and quantitative analysis is based on ADC mapping. An ADC value $\leq 1.25 \times 10^{-3} \mathrm{~mm}^{2} / \mathrm{s}$ was reported to be suitable for differentiating between benign and malignant breast lesions. ${ }^{20,21}$ Quantitative and qualitative analysis of abbreviated unenhanced MRI has been shown to provide higher specificity compared to that of contrast-enhanced MRI. ${ }^{15,24}$

Although unenhanced abbreviated MRI showed comparable diagnostic accuracy, its false-negative rate was higher than that of postcontrast abbreviated MRI. In this study, smaller cancers $(\leq 10 \mathrm{~mm})$ were significantly associated with false-negative findings. Several studies have reported on the drawbacks of DWI MRI. DCIS shows less diffusion impedance, as reflected by higher ADC values, compared with invasive carcinomas, ${ }^{28}$ which might cause relatively low tumour conspicuity on DWI MRI. Previous studies also reported that smaller cancers, specifically $\leq 10$ to $12 \mathrm{~mm}$, were harder to detect. ${ }^{16,17,20,21}$ The typical DWI MRI axial-in-plane spatial resolution $\left(2 \times 2 \mathrm{~mm}^{2}\right)$ and section thickness (3-5 $\mathrm{mm})$ are thought to lead to a marked partial volume effect for small lesions, as well as possible concealment by a susceptibility artefact such as adjacent biopsy marker clips, which is more pronounced on DWI MRI than on conventional T1WI and T2WI. ${ }^{27}$

Malignant lesions with high water content can also be missed because of their high ADC values. Such lesions include mucinous carcinoma and triple-negative cancer with extensive necrosis. ${ }^{28,29}$ One lesion, originally considered to be benign, was mucinous cancer showing a high ADC value and high signal intensity on T2WI (Figure 4).
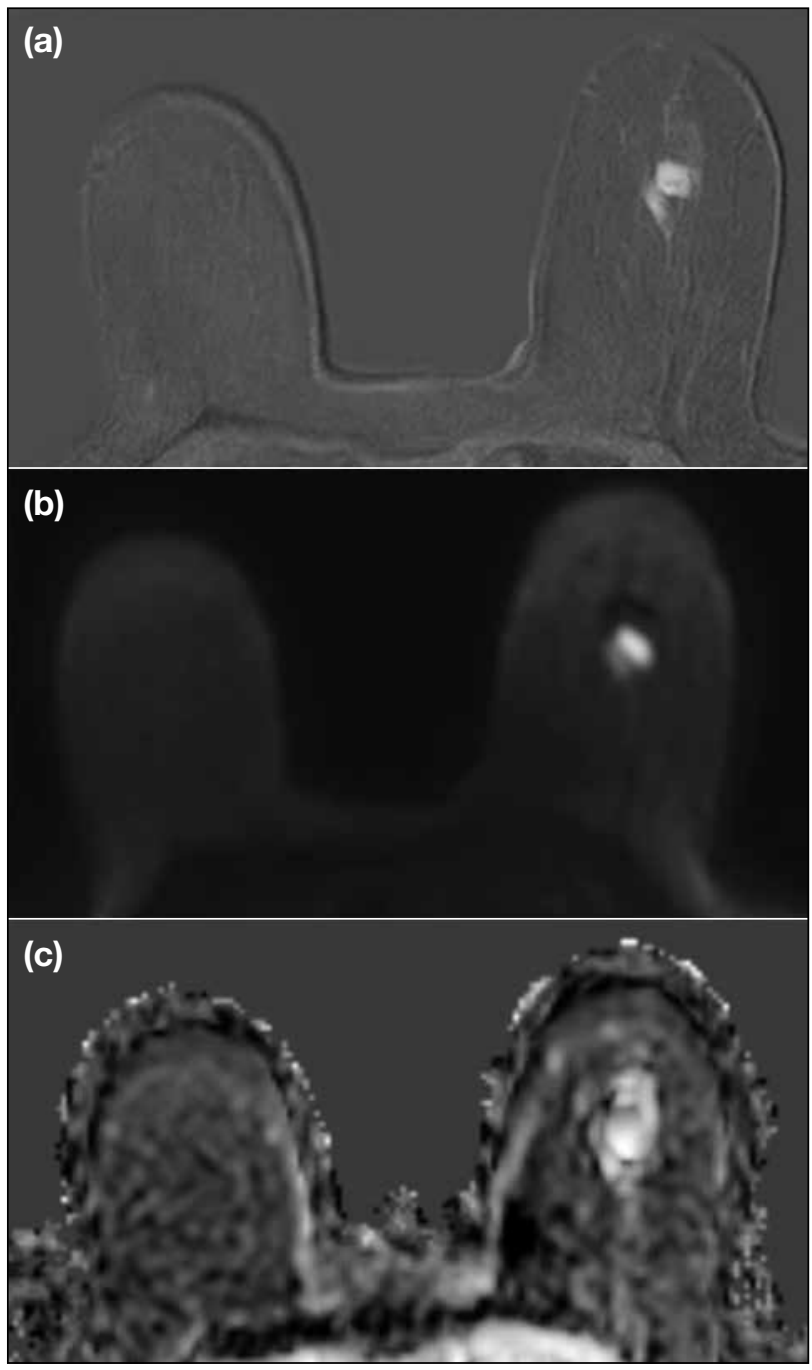

Figure 4. An 82-year-old woman with mucinous carcinoma in the left breast. The lesion was originally considered benign on unenhanced abbreviated magnetic resonance imaging. There was an enhancing mass in left breast on (a) a postcontrast image. The mass showed high signal intensity on both (b) diffusion-weighted imaging and (c) apparent diffusion coefficient map.

DWI MRI shows high accuracy for detecting breast cancer without contrast. In addition, DWI MRI is not affected by breast density, menopausal status or timing during the menstrual cycle, which impede the detection of breast lesions on mammography or FDP MRI. ${ }^{30-32}$ Most of these studies were focused on quantitative DWI MRI, which requires specific software for obtaining an ADC value. However, some institutions do not implement such a protocol. The qualitative analysis of DWI MRI can be performed without a specific protocol by visual assessment. This analysis could reduce reading times, compared with quantitative DWI MRI. A previous study 
compared the detectability of postcontrast abbreviated MRI and unenhanced abbreviated DWI MRI without ADC mapping; they reported that the detectability of DWI MRI was comparable to that of postcontrast abbreviated MRI. ${ }^{24}$ We compared the diagnostic accuracies of quantitative and qualitative analyses of DWI MRI. The sensitivity and specificity of quantitative analysis were not significantly higher than those of qualitative analysis. DWI MRI could be an alternative screening tool for breast cancer.

This study has limitations. First, it was a retrospective study. This design may lead to selection bias. Second, all patients were already known to have breast cancer and underwent breast MRI in a clinical setting. The study population is not representative of patients with breast cancer in the general population. Third, we did not acquire DWI MRI with the use of advanced techniques. We used classic diffusion-weighted 3.0-T MRI with a conventional single-shot echo planar imaging-based sequence. ${ }^{19}$ At $3.0 \mathrm{~T}$, visibility of the lesion on DWI MRI is substantially improved compared with $1.5 \mathrm{~T}$, ${ }^{33}$ but image artefacts are twice as strong. Clinical DWI is based on single-shot echo planar imaging, which is prone to image artefacts. ${ }^{34}$ DWI MRI based on readoutsegmented echo planar imaging was introduced to reduce geometric distortions, image blurring, and ghosting artefacts at $3.0 \mathrm{~T}^{35,36}$

\section{CONCLUSION}

In conclusion, the diagnostic performance of an abbreviated DWI MRI for detecting breast cancers was comparable to that of a postcontrast abbreviated MRI protocol. The DWI protocol might be useful for screening breast MRI in high-risk populations; however, further validation is needed to demonstrate the feasibility of DWI as a breast screening tool.

\section{REFERENCES}

1. Oeffinger KC, Fontham ET, Etzioni R, Herzig A, Michaelson JS, Shih YC, et al. Breast cancer screening for women at average risk: 2015 guideline update from the American Cancer Society. JAMA 2015;314:1599-614.

2. Kriege M, Brekelmans CT, Boetes C, Besnard PE,Zonderland HM, Obdeijn IM, et al. Efficacy of MRI and mammography for breastcancer screening in women with a familial or genetic predisposition. N Engl J Med. 2004;351:427-37.

3. Kuhl C, Weigel S, Schrading S, Arand B, Bieling H, König R, et al. Prospective multicenter cohort study to refine management recommendations for women at elevated familial risk of breast cancer: the EVA trial. J Clin Oncol. 2010;28:1450-7.

4. Sippo DA, Burk KS, Mercaldo SF, Rutledge GM, Edmonds C, Guan Z, et al. Performance of screening breast MRI across women with different elevated breast cancer risk indications. Radiology. 2019;292:51-9.

5. Kuhl CK, Schrading S, Strobel K, Schild HH, Hilgers RD, Bieling HB. Abbreviated breast magnetic resonance imaging (MRI): first postcontrast subtracted images and maximum-intensity projection - a novel approach to breast cancer screening with MRI. J Clin Oncol. 2014;32:2304-10.

6. Grimm LJ, Soo MS, Yoon S, Kim C, Ghate SV, Johnson KS. Abbreviated screening protocol for breast MRI: a feasibility study. Acad Radiol. 2015;22:1157-62.

7. Harvey SC, Di Carlo PA, Lee B, Obadina E, Sippo D, Mullen L. An abbreviated protocol for high-risk screening breast MRI saves time and resources. J Am Coll Radiol. 2016;13:R74-80.

8. Moschetta M, Telegrafo M, Rella L, Stabile Ianora AA, Angelelli G. Abbreviated combined MR protocol: a new faster strategy for characterizing breast lesions. Clin Breast Cancer. 2016;16:207-11.

9. Machida Y, Shimauchi A, Kanemaki Y, Igarashi T, Harada M, Fukuma E. Feasibility and potential limitations of abbreviated breast MRI: an observer study using an enriched cohort. Breast Cancer. 2017;24:411-9.

10. Panigrahi B, Mullen L, Falomo E, Panigrahi B, Harvey S. An abbreviated protocol for high-risk screening breast magnetic resonance imaging: impact on performance metrics and BI-RADS assessment. Acad Radiol. 2017;24:1132-8.

11. Chhor CM, Mercado CL. Abbreviated MRI protocols: wave of the future for breast cancer screening. AJR Am J Roentgenol. 2017;208:284-9.

12. Gulani V, Calamante F, Shellock FG, Kanal E, Reeder SB, International Society for Magnetic Resonance in Medicine. Gadolinium deposition in the brain: summary of evidence and recommendations. Lancet Neurol. 2017;16:564-70.

13. Partridge SC, Mullins CD, Kurland BF, Allain MD, DeMartini WB, Eby PR, et al. Apparent diffusion coefficient values for discriminating benign and malignant breast MRI lesions: effects of lesion type and size. AJR Am J Roentgenol. 2010;194:1664-73.

14. Zhang L, Tang M, Min Z, Lu J, Lei X, Zhang X. Accuracy of combined dynamic contrast-enhanced magnetic resonance imaging and diffusion-weighted imaging for breast cancer detection: a metaanalysis. Acta Radiol. 2016;57:651-60.

15. Chen X, Li WL, Zhang YL, Wu Q, Guo YM, Bai ZI. Meta-analysis of quantitative diffusion-weighted MR imaging in the differential diagnosis of breast lesions. BMC Cancer. 2010;10:693.

16. Kazama T,Kuroki Y,Kikuchi M,Sato Y,NagashimaT,Miyazawa Y, et al. Diffusion-weighted MRI as an adjunct to mammography in women under 50 years of age: an initial study. J Magn Reson Imaging. 2012;36:139-44.

17. Kang JW, Shin HJ, Shin KC, Chae EY, Choi WJ, Cha JH, et al. Unenhanced magnetic resonance screening using fused diffusionweighted imaging and maximum-intensity projection in patients with a personal history of breast cancer: role of fused DWI for postoperative screening. Breast Cancer Res Treat. 2017;165:11928.

18. Kim JY, Kim JJ, Lee H, Lee JW, Lee NK, Nam KJ, et al. Diffusionweighted MRI of estrogen receptor-positive, HER2-negative, node-negative breast cancer: association between intratumoral heterogeneity and recurrence risk. Eur Radiol. 2020;30:66-76.

19. Kim JY, Kim JJ, Lee H, Kang T, Park H. Diffusion-weighted imaging of invasive breast cancer: relationship to distant metastasis-free survival. Radiology. 2019;291:300-7.

20. Baltzer PA, Bickel H, Spick C, Wengert G, Woitek R, Kapetas P, et al. Potential of noncontrast magnetic resonance imaging with diffusion-weighted imaging in characterization of breast lesions: intraindividual comparison with dynamic contrast-enhanced 
magnetic resonance imaging. Invest Radiol. 2018;53:229-35.

21. Pinker K, Moy L, Sutton EJ, Mann RM, Weber M, Thakur SB, et al. Diffusion-weighted imaging with apparent diffusion coefficient mapping for breast cancer detection as a stand-alone parameter: comparison with dynamic contrast-enhanced and multiparametric magnetic resonance imaging. Invest Radiol. 2018;53:587-95.

22. Chen SQ, Huang M, Shen YY, Liu CL, Xu CX. Application of abbreviated protocol of magnetic resonance imaging for breast cancer screening in dense breast tissue. Acad Radiol. 2017;24:31620.

23. Choi BH, Choi N, Kim MY, Yang JH, Yoo YB, Jung HK. Usefulness of abbreviated breast MRI screening for women with a history of breast cancer surgery. Breast Cancer Res Treat. 2018;167:495-502.

24. Yamada T, Kanemaki Y, Okamoto S, Nakajima Y. Comparison of detectability of breast cancer by abbreviated breast MRI based on diffusion-weighted images and postcontrast MRI. Jpn J Radiol. 2018;36:331-9.

25. Shin HJ, Chae EY, Choi WJ, Ha SM, Park JY, Shin KC, et al. Diagnostic performance of fused diffusion-weighted imaging using unenhanced or postcontrast T1-weighted MR imaging in patients with breast cancer. Medicine (Baltimore). 2016;95:e3502.

26. Choi SY, Chang YW, Park HJ, Kim HJ, Hong SS, Seo DY. Correlation of the apparent diffusion coefficiency values on diffusion-weighted imaging with prognostic factors for breast cancer. Br J Radiol. 2012;85:e474-9.

27. Le Bihan D, Poupon C, Amadon A, Lethimonnier F. Artifacts and pitfalls in diffusion MRI. J Magn Reson Imaging. 2006;24:478-88.

28. Woodhams R, Matsunaga K, Kan S, Hata H, Ozaki M, Iwabuchi K, et al. ADC mapping of benign and malignant breast tumors. Magn Reson Med Sci. 2005;4:35-42.

29. Youk JH, Son EJ, Chung J, Kim JA, Kim EK. Triple-negative invasive breast cancer on dynamic contrast-enhanced and diffusionweighted MR imaging: comparison with other breast cancer subtypes. Eur Radiol. 2012;22:1724-34.

30. Hahn SY, Ko ES, Han BK, Lim Y, Gu S, Ko EY. Analysis of factors influencing the degree of detectability on diffusion-weighted MRI and diffusion background signals in patients with invasive breast cancer. Medicine (Baltimore). 2016;95:e4086.

31. Horvat JV, Durando M, Milans S, Patil S, Massler J, Gibbons G, et al. Apparent diffusion coefficient mapping using diffusion-weighted MRI: impact of background parenchymal enhancement, amount of fibroglandular tissue and menopausal status on breast cancer diagnosis. Eur Radiol. 2018;28:2516-24.

32. Iacconi C, Thakur SB, Dershaw DD, Brooks J, Fry CW, Morris EA. Impact of fibroglandular tissue and background parenchymal enhancement on diffusion weighted imaging of breast lesions. Eur J Radiol. 2014;83:2137-43.

33. Matsuoka A, Minato M,Harada M, Kubo H, Bandou Y, Tangoku A, et al. Comparison of 3.0-and 1.5-tesla diffusion-weighted imaging in the visibility of breast cancer. Radiat Med. 2008;26:15-20.

34. Yeom KW, Holdsworth SJ, Van AT, Iv M, Skare S, Lober RM, et al. Comparison of readout-segmented echo-planar imaging (EPI) and single-shot EPI in clinical application of diffusionweighted imaging of the pediatric brain. AJR Am J Roentgenol. 2013;200:W437-43.

35. Bogner W, Pinker-Domenig K, Bickel H, Chmelik M, Weber M, Helbich TH, et al. Readout-segmented echo-planar imaging improves the diagnostic performance of diffusion-weighted MR breast examinations at 3.0 T. Radiology. 2012;263:64-76.

36. Bogner W, Gruber S, Pinker K, Grabner G, Stadlbauer A, Weber M, et al. Diffusion-weighted MR for differentiation of breast lesions at 3.0 T: how does selection of diffusion protocols affect diagnosis? Radiology. 2009;253:341-51. 